\title{
Three-dimensional measurement of internal structure in frozen food materials by cryogenic microtome imaging system
}

\author{
Do, G. ${ }^{\text {a*}}$; Sase, S. ${ }^{\text {a }}$ Bae, Y. ${ }^{\text {b; Maeda, T. }}{ }^{\text {; }}$ Ueno, S. ${ }^{\text {d }}$ and Araki, T. ${ }^{\text {e }}$ \\ ${ }^{\text {a }}$ College of Bioresource Science, Nihon University, Kanagawa, Japan \\ b College of Life Science and Natural Resources, Sunchon National University, Jeollanam-do, Korea \\ ${ }^{\mathrm{c}}$ Nisshin Foods INC., Tokyo, Japan \\ ${ }^{\mathrm{d}}$ Faculty of Education, Saitama University, Saitama, Japan \\ ${ }^{\mathrm{e}}$ Graduate School of Agricultural and Life Sciences, The University of Tokyo, Tokyo, Japan
}

*E-mail of the corresponding author: dogabsoo@nihon-u.ac.jp

\begin{abstract}
The objective of this work was to establish a three-dimensional measuring method for the size, morphology and distribution of internal structure such as ice crystals, bubbles and solids content within an ice cream sample by using a cryogenic microtome spectral imaging system (CMtSIS). The 3-D images of ice crystals, bubbles and milk solids were recognized by reconstructing the circles in 2-D images into 3-D spheres; and the Overrun by Volume (ORV) was obtained by incorporating the area of bubbles on integrated image and the volume of bubbles in the 3-D image.
\end{abstract}

Keywords: Ice crystal, Bubble, Internal structure, Spectral imaging, Micro- to macro-scale, Freeze-Drying 


\section{Introduction}

Most food materials are composed of limited structural elements such as a solid content, bubble and ice crystal. The internal structure in frozen food materials undergoes metamorphic changes due to recrystallization process which increases the size of ice crystals during frozen storage. The size and shape of internal structure formed in a frozen material strongly influence the final quality as well as the freezing rate or heat flux and the direction of heat flow within the material during freezing. For a proper understanding of the behavior of heat or mass transfer in frozen food materials and the behavior of freeze-drying, knowledge of the internal structure of frozen food materials is required. Ice cream is considered as a complex multiphase system consisting of bubbles, ice crystals and milk solids, and the final quality of which is influenced by the correlation between the size, morphology and distribution of the multiphase formed during the manufacturing process. Attempts have been made to observe bubbles and ice crystals on the cross sections of ice cream by using an optical microscope [1] or by indirect methods using SEM [2] or Cryo-SEM [3]. The SEM and Cryo-SEM allow the identification of morphological difference between bubbles and ice crystals by the micro asperity of a sublimated sample surface, but it is difficult to determine whether a sublimated seam is caused by an ice crystal or by a bubble. Furthermore, the measurement of bubble size by traditional methods would result in misleading numbers due to limitations in observing the entire volume of a bubble. Different cross sections would provide different diameters for the same bubble. And the values of the diameters reported by traditional methods might not correlate well with the final quality of ice cream since the measurements on bubbles, ice crystals and milk solids were taken from separate samples. X-ray micro-computed tomography (X-ray $\mu \mathrm{CT}$ ) has been applied to three-dimensional (3-D) structural elements of frozen food materials [4,5]. It is based on the contrast in X-ray images generated by differences in X-ray attenuation arising from differences in the density of material within a sample. Also, frozen food materials that contain water are freeze-dried to clarify density contrast. The objective of this work was to establish a 3-D measuring method for the size, morphology and distribution of internal structure such as ice crystals, bubbles and solids content within ice cream samples prepared at three different overrun levels.

\section{Experimental Equipment and Materials}

\subsection{Cryogenic microtome spectral imaging system}

The CMtSIS [6] consists of a microtome unit, an automatic high-precision XY stage, an image acquisition unit (visible, fluorescence and spectroscopic), and a 3-D image processor (Fig. 1). The CMtSIS allows consecutive image acquisition of the cross sections of a frozen sample exposed by multi-slicing with a minimum thickness of $0.25 \mu \mathrm{m}$. The temperature of the heat exchanger is controlled from room temperature to $-160^{\circ} \mathrm{C}$ by regulating the flow 
rate of liquid nitrogen and the power to an electric heater installed in the heat exchanger. Microscale and macroscale images can be obtained with a highpower objective lens of a microscope installed on the automatic highprecision XY stage (ALD-106-H1P, Chuo Precision Industrial Co., Ltd., Japan) that can be mechanically adjusted with a resolution of $6 \mu \mathrm{m}$ within a maximum area of $60 \times 60 \mathrm{~mm}^{2}$. The image of each cross section is captured with a highly sensitive CCD and an NIR camera, and is recorded by the 3-D image processor. A 3D image is reconstructed from a series of consecutively acquired 2-D images.

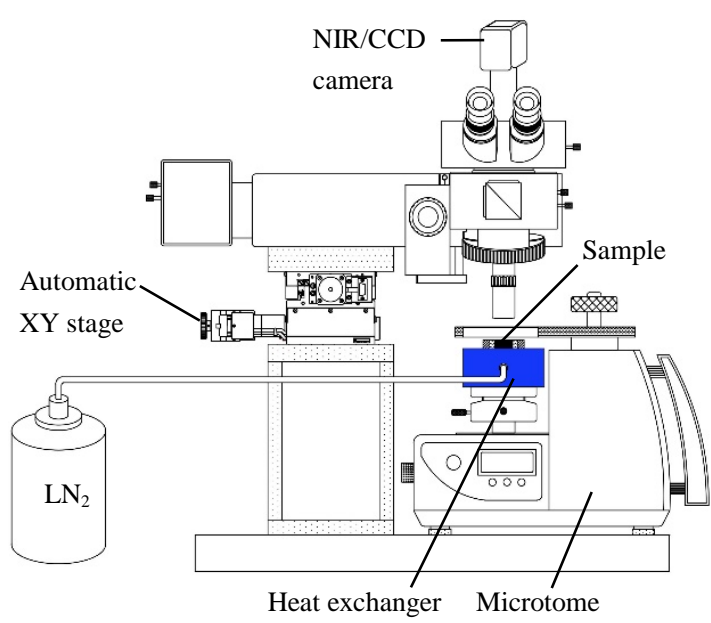

Fig. 1 Schematic diagram of cryogenic microtome spectral imaging system (CMtSIS).

\subsection{Materials and methods}

A lump of vanilla ice cream mix (from ZAO, Yamada milk Co., Ltd. Miyagi, Japan) with a formulation of $8 \%$ milk fat, $10 \%$ milk solids-not-fat, sugar, honey, stabilizer and an emulsifier was taken from a commercially available soft ice cream. Ice cream samples at three different overrun levels (low, medium, and high) were produced by an automatic batch preezer (191 P/SP/N, Carpigiani group, bologna, Italy). Ice cream agglomerate shaped into a cylindrical sample with a diameter of $10 \mathrm{~mm}$ and a height of $10 \mathrm{~mm}$ was shock frozen in liquid nitrogen. The sample was embedded in an optimal cutting temperature (OCT) compound and a ring of dry ice. The sample, OCT compound, and the ring of dry ice fixed on the heat exchanger were sliced together, and cross-sectional images were then captured by the CCD camera through a microscope. Bubbles, ice crystals and milk solids in the ice cream sample were identified from the CMtSIS iamges by the microscale to macroscale measurement method previously reported by Do et al. [6].

\section{Results and Disussion}

\subsection{Overrun by weight}

Overrun is the industrial term for the amount of air added to frozen dessert products, and the percent overrun of ice cream by weight can be calculated by the following equation [7]: 
Three-dimensional Measurement of Internal Structure in Frozen Food Materials by Cryogenic Microtome Imaging System

$$
\% \text { Overrun by weight }(\mathrm{OR})=\frac{W t . \text { of mix }-W t . \text { of same vol.of ice cream }}{W t . \text { of same vol.of ice cream }} \times 100
$$

The overrun of commercial ice cream can range from $24 \%$ (super-premium) to 94\% (light and fluffy). Percent overrum by weight was measured for the ice cream samples prepared at three overrun levels and the mean values were $11.5 \%$, $44.0 \%$ and $73.8 \%$ for low, medium, and high overrun levels, respectively (Table $1)$.

\subsection{Measurement of bubble}

A total of 36 image frames was obtained from a sliced surface by using the CCD camera, the $\times 50$ magnification lens, and the automatic high-precision XY stage. Each frame covered an area of $194 \times 154 \mu^{2}$. The 36 frames were integrated into a single image by a 3-D image processor. Fig. 2 shows an integrated raw image and a binary image representing the boundaries of identified bubbles covering a measurement area of $1164 \times 924 \mu^{2}$ with a total of $7800 \times 6180$ pixels. Fig. 3 shows the distribution of equivalent circle diameters of the bubbles in the sample of $11.5 \%$ OR (low overrun). A total of 480 bubbles was recognized from the integrated image: the equivalent circle diameters ranged from 1.1 to $101.9 \mu \mathrm{m}$ with a mean of $24.8 \mu \mathrm{m}$, and the measured areas ranged from 0.9 to $8144.9 \mu \mathrm{m}^{2}$ with a mean of $637.8 \mu \mathrm{m}^{2}$. Fig. 4 shows the distribution of equivalent circle diameters of the bubbles found in the sample of $44.0 \%$ OR (medium overrun). A total of 1988 bubbles was recognized from

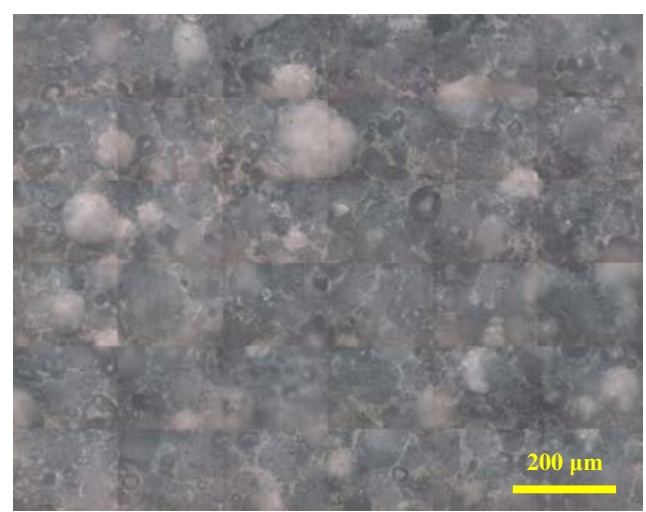

a) Raw iamge

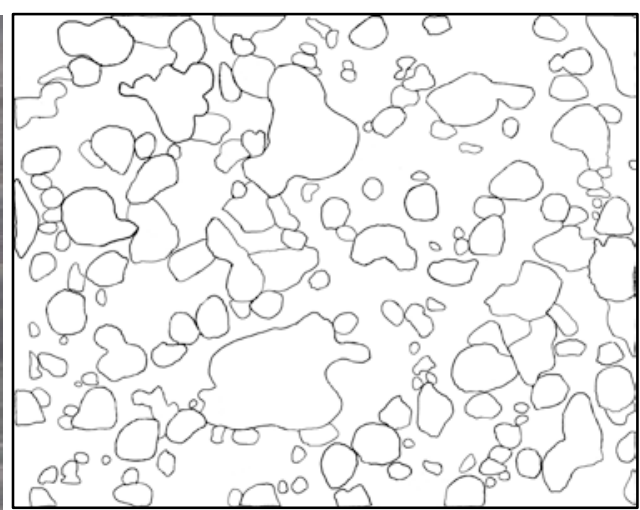

b) Boundaries of bubbles

Fig. 2 Binarization of an integrated image to identify the bubbles in an ice cream sample. 


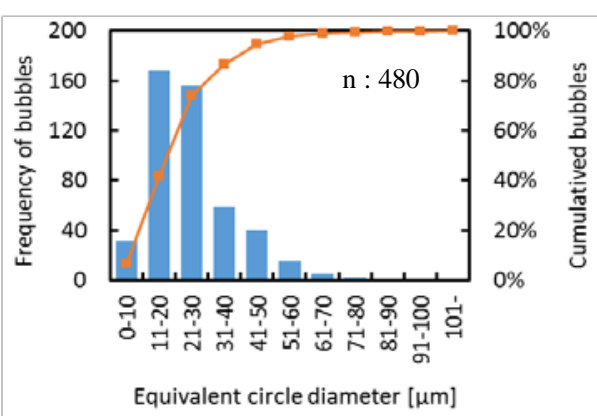

Fig. 3 Equivalent circle diameter of bubbles in the sample of $11.6 \% \mathrm{OR}$.

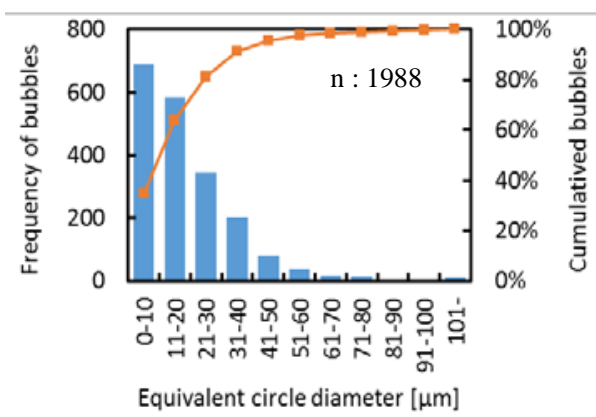

Fig. 4 Equivalent circle diameter of bubbles in the sample of $44.0 \% \mathrm{OR}$.

the integrated image: the equivalent circle diameters ranged from 1.1 to $140.4 \mu \mathrm{m}$ with a mean of $25.8 \mu \mathrm{m}$, and the areas ranged from 0.9 to $15479.8 \mu \mathrm{m}^{2}$ with a mean of 749.0 $\mu \mathrm{m}^{2}$. Fig. 5 shows the distribution of the equivalent circle diameters of the bubbles in the high overrun sample (73.8\% OR). A total of 692 bubbles was recognized from the integrated image: the equivalent circle diameters ranged from 1.1 to $248.2 \mu \mathrm{m}$ with a mean of $40.3 \mu \mathrm{m}$, and the areas ranged from

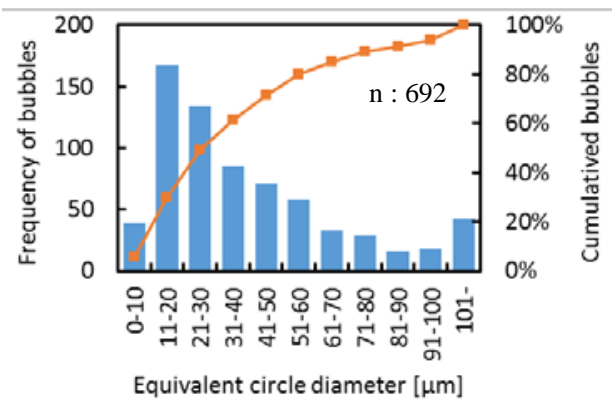

Fig. 5 Equivalent circle diameter of bubbles in the sample of $73.8 \% \mathrm{OR}$. 0.9 to $48362.0 \mu \mathrm{m}^{2}$ with a mean of $2035.9 \mu \mathrm{m}^{2}$. The actual size of the integrated images was $1164 \times 924 \mu^{2}$ and from these images the range of the areas of the recognized bubbles was determined to be from microscale of 0.9 to macroscale of $48362.0 \mu \mathrm{m}^{2}$.

\subsection{Overrun by volume}

The CMtSIS has functions to capture the cross-sections exposed by multi-slicing of a frozen sample with a minimum thickness of $1 \mu \mathrm{m}$ and to reconstruct a 3-D image utilizing a series of consecutively acquired 2-D images. The volume rendering method was employed as the optimum one for the system to measure internal structures. Using this method the volume can be determined from the surface areas of quantitative 2-D information. Overrun can be determined as the percentage increase in volume or area of the mix that occurs as a result of air addition, and can also be calculated by the following equation [7]:

$$
\% \text { Overrun by volume (ORV) }=\frac{\text { Vol.of ice cream }- \text { Vol.of mix used }}{\text { Vol.of mix used }} \times 100
$$


Fig. 6 compares the OR values calculated from the weight measurements and the ORV values obtained from the area of bubbles on integrated image analysis using the CMtSIS for the ice cream samples prepared at three overrun levels. The means of obtained ORV values were $10.5 \%, 42.8 \%$, and $77.7 \%$ for low, medium, and high overrun levels, respectively; whereas the corresponding OR measurements were $11.5 \%$, $44.0 \%$, and $73.8 \%$. Thus, the measurement accuracy of the CMtSIS was verified in

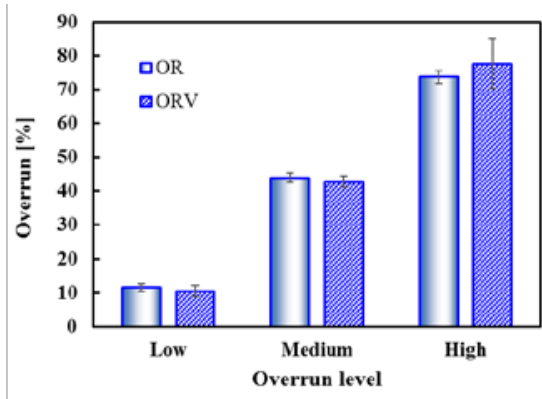

Fig. 6 OR and ORV for different overrun levels. estimating the percent overrun by volume for frozen ice cream samples.

\subsection{Three-dimensional measurement of the internal structure of ice cream}

An ice cream sample of $12.7 \%$ OR was prepared to measure the 3-D internal structure. Although the bubbles, ice crystals and milk solids could be recognized from an original 2-D image (Fig. 7) by visual evaluation, it was difficult to obtain a binary image of these only by applying automatic thresholding techniques. Therefore, the boundaries of individual bubbles, ice crystals and milk solid were traced manually on a digitizing board (Cintiq 21UXTZ-2100, WACOM Ltd, Japan) as shown in the upper row of Fig. 7. After extracting the pixels within the traced boundaries and obtaining binary images, the volumes of each bubble and ice crystal were calculated to represent the morphology. The 3-D images of bubbles, ice crystals and milk solids were reconstructed using 100 cross-sectional images (slicing thickness: $4 \mu \mathrm{m}$ ). The 3-D images of bubbles, ice crystals and milk solids (the lower row of Fig. 7) were reconstructed based on the binary images. The dimension of each 3-D image was $260 \mu \mathrm{m}$ in length, $206 \mu \mathrm{m}$ in width and $400 \mu \mathrm{m}$ in height. By using an image processing software (TRI/3D VOL, Ratoc Ltd, Japan), the volumes of each bubble and ice crystal were calculated from the 3-D images. A total of 1178 bubbles was recognized by reconstructing the circles in the 2-D images into 3-D spheres and their volumes ranged from $0.125 \mathrm{~mm}^{3}$ to 490.421 $\mathrm{mm}^{3}$ with a mean of $2.342 \mu \mathrm{m}^{3}$ as shown in Fig. 8. Furthermore, an ORV of $14.8 \%$ was obtained by incorporationg the volume of bubbles in the 3-D image for the ice cream sample of $12.7 \%$ OR. A total of 186 ice crystals were recognized by reconstructing the circles in the 2-D images into 3-D spheres and their volumes ranged from $0.125 \mathrm{~mm}^{3}$ to $5493.684 \mathrm{~mm}^{3}$ with a mean of $34.483 \mathrm{~mm}^{3}$ as shown in Fig. 9. By observing the 3-D images (Fig. 7-b, c), it is recognized that the bubbles are evenly distributed in the ice cream sample, but the ice crystals are less evenly distributed. Milk solids are observed in the form of a retinal structure circumscribing bubbles and ice crystals as shown in Fig. 7-a.d. The bubbles are observed within ice crystals and milk solids. 
Measurement of bubbles is difficult when using SEM or Cryo-SEM because of sublimation at the sample surface. Ice crystals and bubbles within a sample can be resolved by the vacant spaces left after freeze-drying. Since all vacant spaces are treated as ice crystals and bubbles in the analysis of a freeze-drying process, some questions may be raised to the identification of ice crystals and bubbles. Therefore, a 3-D measuring method for the size, morphology and distribution of internal structure such as ice crystals, bubbles and solids content within an ice cream sample was established in this research by using the CMtSIS.

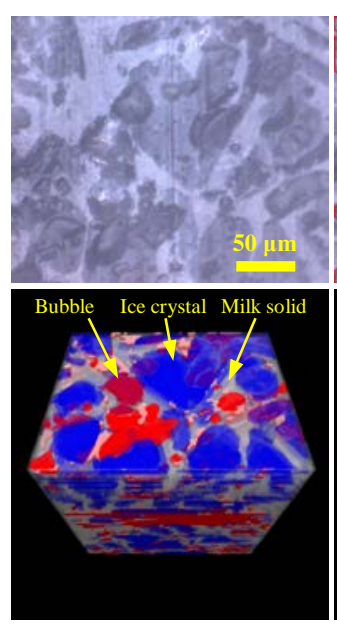

a) Ice cream
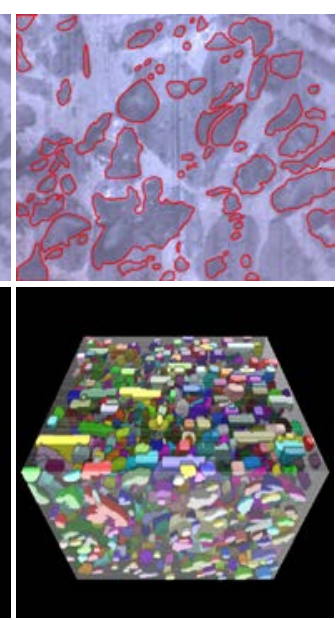

b) Bubble
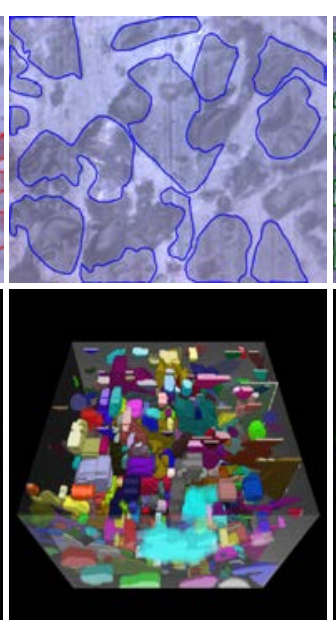

c) Ice crystal
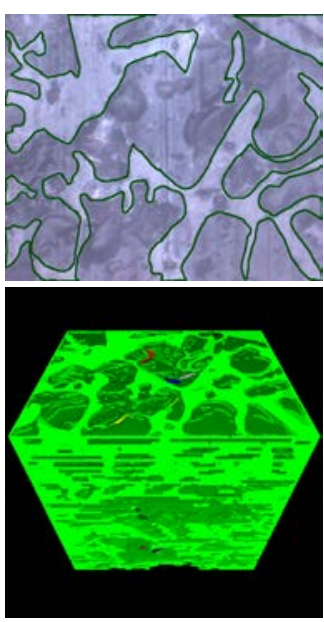

d) Milk solid

Fig. 7 Morphology and distribution of internal structure of ice cream.

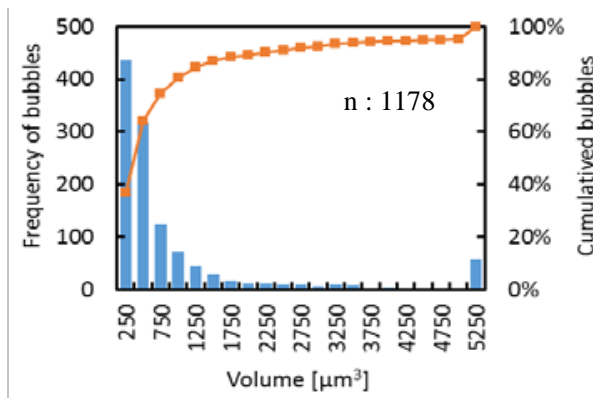

Fig. 8 Distribution of volume of bubbles.

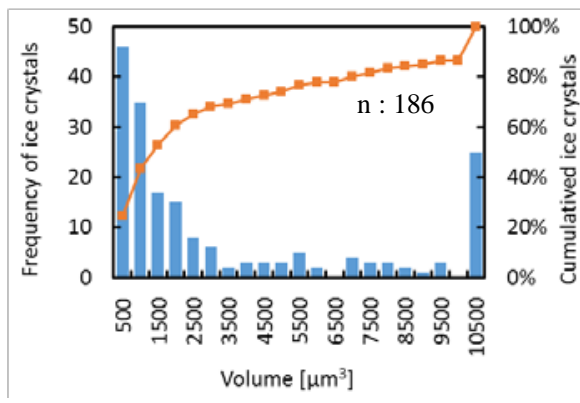

Fig. 9 Distribution of volume of ice crystals.

\section{Conclusions}

A novel technique was developed for the detection of 3-D internal structure of ice cream samples produced at different overrun levels and its high accuracy was verified by using the CMtSIS. The following conclusions were reached: 
1. The actual size of the integrated images was $1164 \times 924 \mu \mathrm{m}^{2}$ and from these images the range of the areas of the recognized bubbles was determined to be from microscale of 0.9 to macroscale of $48362.0 \mu \mathrm{m}^{2}$.

2. The means of obtained ORVs were $10.5 \%, 42.8 \%$, and $77.7 \%$, whereas the means of the measured ORs were $11.5 \%, 44.0 \%$ and $73.8 \%$ for the ice cream samples produced at three overrun levels (low, medium, and high, respectively).

3. The estimated volumes of bubbles ranged from $0.125 \mathrm{~mm}^{3}$ to $490.421 \mathrm{~mm}^{3}$ with a mean of $2.342 \mu \mathrm{m}^{3}$, and those for ice crystals ranged from $0.125 \mathrm{~mm}^{3}$ to $5493.684 \mathrm{~mm}^{3}$ with a mean of $34.483 \mathrm{~mm}^{3}$ in the 3-D image for the ice cream sample of $12.7 \%$ OR.

4. The ORV of $14.8 \%$ was obtained by incorporating the volume of bubbles in the $3-\mathrm{D}$ image in the case of the ice cream sample of $12.7 \%$ OR.

\section{Acknowledgment}

This work was supported by a Kakenhi Grant-in-Aid (No. 17K00826) from the Japan Society for the Promotion of Science (JSPS).

\section{References}

[1] Chang Y., Hartel R.W. (2002), Development of air cells in a batch ice cream freezer, Journal of Food Engineering, 55, pp71-78

[2] Eisner M. D., Wildmoser H., Windhab E. J. (2005), Air cell micro structuring in a high viscous ice cream matrix, Colloids and Surfaces A: Physicochem. Eng. Aspects, 263, pp390-399.

[3] Goff H.D. Verespej E., Smith A.K. (1999), A study of fat and air structures in ice cream, International Dairy Journal, 9, pp817-829.

[4] Zhao, Y.; Takhar, P. S. Micro X-ray computed tomography and image analysis of frozen potatoes subjected to freeze-thaw cycles, LWT - Food Science and Technology, 2017, 79, 278-286.

[5] Jin, J.; Yurkow, E. J.; Adler, D.; Lee, T. Improved freeze drying efficiency by ice nucleation proteins with ice morphology modification, Food Research International, 2018, 106, 90-97.

[6] Do, G.; Sase, S.; Bae, Y.; Maeda, T.; Ueno, S.; Araki, T. Microscale to Macroscale Measurement of Bubbles in Frozen Materials with Cryogenic Microtome Spectral Imaging System, Japan Journal of Food Engineering, 2017, 18(3), 125-132.

[7] Goff, H. D.; Hartel, R.W. Ice cream, seventh edition; Springer: New York, 2013; 184191. 